\title{
THE ANALYSIS OF MULTIDIMENSIONAL ANOMALOUS DIFFUSION EQUATION
}

\section{Bambang Hendriya Guswanto}

Analysis and Algebra Laboratory, Department of Mathematics Faculty of Mathematics and Natural Sciences, Jenderal Soedirman University bambang.guswanto@unsoed.ac.id

\begin{abstract}
We discuss the properties of the fundamental solution of multidimensional anomalous diffusion equation such as symmetric, decay, nonnegative, normality, and bounded in mathematical analysis approach.
\end{abstract}

Keywords. fundamental solution, anomalous diffusion, symmetric, decay, nonnegative, normal.

ABSTRAK. Makalah ini membahas sifat-sifat dari penyelesaian fundamental dari persamaan difusi anomali seperti simetri, luruh, nonnegatifan, dan normal dengan menggunakan pendekatan matematika analisis.

Kata Kunci. Penyelesaian fundamental, difusi anomali, simetrian, luruh, nonnegatif, normal.

\section{INTRODUCTION}

Anomalous diffusion is a diffusion process which has the characteristic that the mean square displacement (MSD) of a particle moving in the process with respect to time $t>0$ follows the pattern

$$
\left\langle x^{2}(t)\right\rangle \sim t^{\alpha}, \alpha>0, \alpha \neq 1 .
$$

When $\alpha=1$, the process is diffusion. If $0<\alpha<1$, the process is called subdiffusion or slow diffusion. This kind of anomalous diffusion is modelled by the equation

$$
\frac{\partial^{\alpha} u}{\partial t^{\alpha}}=D_{\alpha} \Delta u, \quad 0<\alpha<1,
$$

where $u=u(x, t)$ denotes the concentration of the particle at site $x \in \mathbb{R}^{n}$ and time $t>0, D_{\alpha}$ is a subdiffusion coefficient, and $d^{\alpha} / d t^{\alpha}$ is Caputo fractional time derivative defined by 


$$
\frac{d^{\alpha}}{d t^{\alpha}} f(t)=\int_{0}^{t} \frac{(t-\tau)^{-\alpha}}{\Gamma(1-\alpha)} \cdot \frac{d}{d \tau} f(\tau) d \tau, \quad 0<\alpha<1 .
$$

When $\gamma>1$, the process is called superdiffusion or fast diffusion and modelled by the equation

$$
\frac{\partial u}{\partial t}=-C_{\beta}(-\Delta)^{\frac{\beta}{2}} u, \quad 0<\beta<2,
$$

where $C_{\beta}$ is a superdiffusion coefficient and $(-\Delta)^{\beta / 2}$ is called fractional laplacian operator which has the property

$$
\mathcal{F}\left((-\Delta)^{\beta / 2} u\right)(\xi)=|\xi|^{\beta} \mathcal{F}(u)(\xi)
$$

where $\mathcal{F}$ denotes the fourier transform operator defined by

$$
\mathcal{F}(u(s))(\xi)=\int_{0}^{\infty} e^{-s \xi} u(s) d s .
$$

If $\beta=2$, the operator is called Laplacian.

Here, we discuss the properties of the fundamental solution of the general form of both equation, that is

$$
\begin{aligned}
& \frac{\partial^{\alpha} u}{\partial t^{\alpha}}=-(-\Delta)^{\frac{\beta}{2}} u, \quad t>0, \\
& u(x, 0)=\delta(x), \quad x \in \mathbb{R}^{n},
\end{aligned}
$$

where $0<\alpha<1,0<\beta<2$. The properties of the solution discussed here are symmetric, decay, nonnegative, and normal.

This paper is composed of four sections. In the second section, we explain briefly a special function which is called Mittag-Leffler function. We show our main results in the third section. Finally, in the last section, the conclusion of our discussion is given.

\section{PRELIMINARIES}

We here introduce the Mittag-Leffler function defined by 


$$
E_{\alpha, \beta}(z)=\sum_{n=0}^{\infty} \frac{z^{n}}{\Gamma(\alpha n+\beta)}, \alpha, \beta>0, z \in \mathbb{C} .
$$

This function is entire. For $\beta=1$, we set $E_{\alpha, \beta}(z)=E_{\alpha}(z)$, and, for $\alpha=\beta=1$, we have that $E_{\alpha, \beta}(z)$ is nothing but exponential function $e^{z}$.

We next give the asymptotic formulas for the Mittag-Leffler function. For $0<\alpha<2, \beta$ is an arbitrary complex number, and $\mu$ is an arbitrary number such that

$$
\frac{\pi \alpha}{2}<\mu<\min \{\pi, \pi \alpha\},
$$

then, for an arbitrary integer $p \geq 1$, the following hold, those are

$$
\begin{gathered}
E_{\alpha, \beta}(z)=\frac{1}{\alpha} z^{(1-\beta) / \alpha} e^{1 / \alpha}-\sum_{n=1}^{p} \frac{z^{-n}}{\Gamma(\beta-\alpha n)}+O\left(|z|^{-1-p}\right), \\
|z| \rightarrow \infty, \quad|\arg (z)| \leq \mu,
\end{gathered}
$$

and

$$
E_{\alpha, \beta}(z)=-\sum_{n=1}^{p} \frac{z^{-n}}{\Gamma(\beta-\alpha n)}+O\left(|z|^{-1-p}\right), \quad|z| \rightarrow \infty, \quad \mu \leq|\arg (z)| \leq \mu .
$$

The following well known Proposition tell us the application of Mittag-Leffler function to fractional ordinary differential equation.

Proposition 1. Let $\lambda \in \mathbb{C}$ and $f$ be given complex function defined in $(0, \infty)$. If $v:[0, \infty) \rightarrow \mathbb{C}$ is a continuous function solving the fractional ordinary differential equation

$$
\begin{aligned}
\frac{d^{\alpha}}{d t^{\alpha}} f(t) & =\lambda v(t)+f(t), \quad t>0 \\
v(0) & =v_{0},
\end{aligned}
$$

then it is given uniquely by

$$
v(t)=E_{\alpha}\left(\lambda t^{\alpha}\right) v_{0}+\int_{0}^{t}(t-s)^{\alpha-1} E_{\alpha, \alpha}\left(\lambda(t-s)^{\alpha}\right) f(s) d s, t>0 .
$$


For more details concerning the fractional integrals and derivatives and the Mittag-Lefller function, we refer to Podlubny [5].

\section{MAIN RESULTS}

In this section, we study some properties of the fundamental solutions of anomalous diffusion equation : symmetric, decay, nonnegative, and bounded. Let us consider the initial value problem

$$
\begin{gathered}
\frac{\partial^{\alpha} u}{\partial t^{\alpha}}+(-\Delta)^{\beta / 2} u=0, \text { in } \mathbb{R}^{n} \times(0, \infty) \\
u(\cdot, 0)=u_{0}, \text { in } \mathbb{R}^{n}, \\
u(x, t)=0, t>0,|x| \rightarrow \infty,
\end{gathered}
$$

where $0<\alpha<1,0<\beta<2$. We transform the problem into Fourier domain. Then

$$
\begin{gathered}
\frac{\partial^{\alpha}}{\partial t^{\alpha}} \mathcal{F}(u)(k, t)+|k|^{\beta} \mathcal{F}(u)(k, t)=0, \text { in }(0, \infty) \\
\mathcal{F}(u)(k, 0)=\mathcal{F}\left(u_{0}\right)(k) .
\end{gathered}
$$

Therefore, by Proposition 1, we get

$$
\mathcal{F}(u)(k, t)=\mathcal{F}\left(u_{0}\right)(k) E_{\alpha}\left(-|k|^{\beta} t^{\alpha}\right) .
$$

Then, we obtain the fundamental solution to the problem

$$
\begin{aligned}
u(x, t) & =\mathcal{F}^{-1}\left(\mathcal{F}\left(u_{0}\right)(k) E_{\alpha}\left(-|k|^{\beta} t^{\alpha}\right)\right) \\
& =\mathcal{F}^{-1}\left(\mathcal{F}\left(u_{0}\right)(k)\right)(x) * \mathcal{F}^{-1}\left(E_{\alpha}\left(-|k|^{\beta} t^{\alpha}\right)\right)(x, t) \\
& =u_{0}(x) * G_{\alpha, \beta}(x, t) \\
& =\int_{\mathbb{R}^{n}} u_{0}(y) G_{\alpha, \beta}(x-y, t) d y
\end{aligned}
$$

where

$$
\begin{aligned}
G_{\alpha, \beta}(x, t) & =\mathcal{F}^{-1}\left(E_{\alpha}\left(-|k|^{\beta} t^{\alpha}\right)\right)(x, t) \\
& =\frac{1}{(2 \pi)^{n}} \int_{\mathbb{R}^{n}} e^{-i k \cdot x} E_{\alpha}\left(-|k|^{\beta} t^{\alpha}\right) d k
\end{aligned}
$$




$$
\begin{aligned}
& =\frac{1}{(2 \pi)^{n}} \int_{\mathbb{R}^{n}} e^{-i k \cdot x} E_{\alpha}\left(-\left|k t^{\alpha / \beta}\right|^{\beta}\right) d k \\
& =\frac{t^{-n \alpha / \beta}}{(2 \pi)^{n}} \int_{\mathbb{R}^{n}} e^{-i l \cdot\left(x t^{-\alpha / \beta}\right)} E_{\alpha}\left(-|l|^{\beta}\right) d l \\
& =t^{-n \alpha / \beta} K_{\alpha, \beta}\left(x t^{-\alpha / \beta}\right)
\end{aligned}
$$

and

$$
K_{\alpha, \beta}(y)=\frac{1}{(2 \pi)^{n}} \int_{\mathbb{R}^{n}} e^{-i k \cdot y} E_{\alpha}\left(-|k|^{\beta}\right) d k .
$$

When $u_{0}(x)=\delta(x)$, Dirac Delta function, we have that $u(x, t)=G_{\alpha, \beta}(x, t)$, anomalous "Green function". Henceforth, we assume the case.

Next, since $E_{\alpha}\left(-|k|^{\beta}\right)$ is symmetric, $G_{\alpha, \beta}(x, t)$ is also symmetric in space domain. Consider now

$$
S_{N}(k):=\sum_{j=0}^{N} \frac{|k|^{\beta j}}{\Gamma(\alpha j+1)} \rightarrow E_{\alpha}\left(|k|^{\beta}\right) .
$$

We have that $\left\{S_{N}(k)\right\}_{0}^{\infty}$ is non-decreasing and integrable function sequence on a ball $B_{r}(0)$ with the center at the origin and radius $r>0$. By the Monotone Convergence theorem, $E_{\alpha}\left(|k|^{\beta}\right)$ is integrable on $B_{r}(0)$. Since

$$
\left|E_{\alpha}\left(-|k|^{\beta}\right)\right| \leq E_{\alpha}\left(|k|^{\beta}\right),
$$

we get that $\left|E_{\alpha}\left(-|k|^{\beta}\right)\right|$ is also integrable on $B_{r}(0)$.

We now suppose

$$
I(r)=\int_{B_{r}(0)}\left|E_{\alpha}\left(-|k|^{\beta}\right)\right| d k, r>0 .
$$

From [5], we have, for $0<\alpha<1$,

$$
E_{\alpha}(z)=-\sum_{j=1}^{p} \frac{z^{-j}}{\Gamma(1-\alpha j)}+O\left(|z|^{-1-p}\right),|z| \rightarrow \infty, \frac{\alpha \pi}{2}<\arg z<2 \pi-\frac{\alpha \pi}{2} .
$$


Note that $-|k|^{\beta}$ is non-positive real number (a complex number with $\arg =\pi$ ).

Then, for $0<\alpha<1$,

$$
\left|E_{\alpha}\left(-|k|^{\beta}\right)\right|=\left|-\sum_{j=1}^{p} \frac{(-1)^{j}|k|^{-\beta j}}{\Gamma(1-\alpha j)}\right|=\sum_{j=1}^{p} \frac{|k|^{-\beta j}}{\Gamma(1-\alpha j)} \rightarrow 0 \text {, as }|k| \rightarrow \infty .
$$

Then, for $r<s$,

$$
|I(r)-I(s)|=\int_{B_{S}(0) \backslash B_{r}(0)}\left|E_{\alpha}\left(-|k|^{\beta}\right)\right| d k \rightarrow 0, \text { as } r \rightarrow \infty .
$$

It means $I(r)$ is Cauchy sequence, and, hence,

$$
I(r) \rightarrow \int_{\mathbb{R}^{n}}\left|E_{\alpha}\left(-|k|^{\beta}\right)\right| d k<\infty, \text { as } r \rightarrow \infty .
$$

It means $E_{\alpha}\left(-|k|^{\beta}\right) \in L^{1}\left(\mathbb{R}^{n}\right)$. Therefore, we obtain $K_{\alpha, \beta}(x)$ and $G_{\alpha, \beta}(x, t)$, as the inverse of the Fourier transform of $E_{\alpha}\left(-|k|^{\beta}\right)$ and $E_{\alpha}\left(-|k|^{\beta} t^{\alpha}\right)$, respectively, exist. We next use the following theorem to show the decay property of the fundamental solution.

Riemann-Lebesgue Theorem. If $f \in L^{1}\left(\mathbb{R}^{n}\right)$, then

$$
\int_{\mathbb{R}^{n}} e^{-i k \cdot x} f(x) d x \rightarrow 0, \text { as }|x| \rightarrow \infty
$$

By the Riemann-Lebesgue theorem, since $E_{\alpha}\left(-|k|^{\beta}\right) \in L^{1}\left(\mathbb{R}^{n}\right)$ and

$$
K_{\alpha, \beta}(y)=\frac{1}{(2 \pi)^{n}} \int_{\mathbb{R}^{n}} e^{-i k \cdot y} E_{\alpha}\left(-|k|^{\beta}\right) d k,
$$

we have $\lim _{|x| \rightarrow \infty} K_{\alpha, \beta}(x)=0$. Therefore,

$$
\lim _{|x| \rightarrow \infty} G_{\alpha, \beta}(x, t)=\lim _{|x| \rightarrow \infty} t^{-n \alpha / \beta} K_{\beta}\left(x t^{-\alpha / \beta}\right)=0 .
$$


We next check the nonnegativity and normality of $G_{\alpha, \beta}(x, t)$. In [6], it was showed that, for $0 \leq \alpha \leq 1, E_{\alpha}(-z)$ is a completely monotonic function, that is, for $z \in \mathbb{R}, z \geq 0$,

$$
(-1)^{n} \frac{d^{n}}{d x^{n}} E_{\alpha}(-x) \geq 0, n=0,1,2, \cdots .
$$

Therefore, we have $E_{\alpha}\left(-|k|^{\beta}\right) \geq 0$ and, thus, $G_{\alpha, \beta}(x, t) \geq 0$. Furthermore, for $t>0$,

$$
\begin{aligned}
\left\|G_{\alpha, \beta}(\cdot, t)\right\|_{L^{1}\left(\mathbb{R}^{n}\right)} & =\int_{\mathbb{R}^{n}} G_{\alpha, \beta}(x, t) d x \\
& =\int_{\mathbb{R}^{n}} \frac{1}{(2 \pi)^{n}} \int_{\mathbb{R}^{n}} e^{-i k \cdot x} E_{\alpha}\left(-|k|^{\beta} t^{\alpha}\right) d k d x \\
& =\int_{\mathbb{R}^{n}}\left(\frac{1}{(2 \pi)^{n}} \int_{\mathbb{R}^{n}} e^{-i k \cdot x} d x\right) E_{\alpha}\left(-|k|^{\beta} t^{\alpha}\right) d k \\
& =\int_{\mathbb{R}^{n}} \delta(k) E_{\alpha}\left(-|k|^{\beta} t^{\alpha}\right) d k \\
& =E_{\alpha}(0) \\
& =1 .
\end{aligned}
$$

\section{CONCLUSION}

The fundamental solution to the problem

$$
\begin{gathered}
\frac{\partial^{\alpha}}{\partial t^{\alpha}} u(x, t)=-(-\Delta)^{\frac{\beta}{2}} u(x, t), \quad x \in \mathbb{R}^{n}, t>0, \\
u(x, 0)=\delta(x), \quad x \in \mathbb{R}^{n},
\end{gathered}
$$

where $0<\alpha<1,0<\beta<2$ is

$$
u(x, t)=G_{\alpha, \beta}(x, t)
$$

where

$$
G_{\alpha, \beta}(x, t)=\frac{t^{-n \alpha / \beta}}{(2 \pi)^{n}} \int_{\mathbb{R}^{n}} e^{-i k \cdot\left(x t^{-\alpha / \beta}\right)} E_{\alpha}\left(-|k|^{\beta}\right) d k
$$


which has the properties :

(a) symmetric in space: $G_{\alpha, \beta}(x, t)=G_{\alpha, \beta}(-x, t), x \in \mathbb{R}^{n}$;

(b) decay : $\lim _{|x| \rightarrow \infty} G_{\alpha, \beta}(x, t)=0$;

(c) nonnegative : $G_{\alpha, \beta}(x, t) \geq 0, x \in \mathbb{R}^{n}, t>0$;

(d) normal : $\left\|G_{\alpha, \beta}(\cdot, t)\right\|_{L^{1}\left(\mathbb{R}^{n}\right)}=1$.

\section{REFERENCES}

[1] Gorenflo, R., Luchko, Y., Mainardi, F., Wright Function as Scale-Invariant Solutions of the Diffusion-Wave equation, J. Comput. Appl. Math. 118, 175191 (2000).

[2] Mainardi, F., Mura, A., Pagnini, G., The M-Wright Function in TimeFractional Diffusion Process : A Tutorial Survey, International Journal of Differential Equations, 2010.

[3] Mainardi, F., Luchko, Y., Pagnini, G., The Fundamental Solution of the Space-Time Fractional Diffusion Equation, Frac. Calc. Appl. Anal. 4, 153192 (2001).

[4] Peng, J., Li, Kexue, A Note on Property of the Mittag-Leffler Function, J. Math. Anal. Appl. 370, 635-638, 2010.

[5] Podlubny, I., Fractional Differential Equations, Academic Press, San Diego (1999).

[6] Pollard, H., The Completely Monotonic of the Mittag-Leffler Function $E_{a}(-x)$, Bull. Amer. Math. Soc., 54(12), 1115-1116, 1948.

[7] Stankovic, B., On the Function of E. M. Wright, Publications de L'Institut Mathematique, Nouvelle series 10(24), 113-124 (1970). 